\title{
AIDA: Identifying Code Switching in Informal Arabic Text
}

\author{
Heba Elfardy \\ Department of Computer Science \\ Columbia University \\ New York, NY \\ heba@cs.columbia.edu
}

\author{
Mohamed Al-Badrashiny, Mona Diab \\ Department of Computer Science \\ The George Washington University \\ Washington, DC \\ \{badrashiny, mtdiab\}@gwu.edu
}

\begin{abstract}
In this paper, we present the latest version of our system for identifying linguistic code switching in Arabic text. The system relies on Language Models and a tool for morphological analysis and disambiguation for Arabic to identify the class of each word in a given sentence. We evaluate the performance of our system on the test datasets of the shared task at the EMNLP workshop on Computational Approaches to Code Switching (Solorio et al., 2014). The system yields an average token-level $\mathrm{F}_{\beta=1}$ score of $93.6 \%, 77.7 \%$ and $80.1 \%$, on the first, second, and surprise-genre test-sets, respectively, and a tweet-level $\mathrm{F}_{\beta=1}$ score of $4.4 \%, 36 \%$ and $27.7 \%$, on the same test-sets.
\end{abstract}

\section{Introduction}

Most languages exist in some standard form while also being associated with informal regional varieties. Some languages exist in a state of diglossia (Ferguson, 1959). Arabic is one of those languages comprising a standard form known as Modern Standard Arabic (MSA), that is used in education, formal settings, and official scripts; and dialectal variants (DA) corresponding to the native tongue of Arabic speakers. While these variants have no standard orthography, they are commonly used and have become pervasive across web-forums, blogs, social networks, TV shows, and normal daily conversations. Arabic dialects may be divided into five main groups: Egyptian (including Libyan and Sudanese), Levantine (including Lebanese, Syrian, Palestinian and Jordanian), Gulf, Iraqi and Moroccan. Sub-dialectal variants also exist within each dialect (Habash, 2010). Speakers of a specific Arabic Dialect typically code switch between their dialect and
MSA, and less frequently between different dialects, both inter and intra-sententially. The identification and classification of these dialects in diglossic text can enhance semantic predictability.

In this paper we modify an existing system AIDA (Elfardy and Diab, 2012b), (Elfardy et al., 2013) that identifies code switching between MSA and Egyptian DA (EDA). We apply the modified system to the datasets used for evaluating systems participating at the EMNLP Workshop on Computational Approaches to Linguistic Code Switching. ${ }^{1}$

\section{Related Work}

Dialect Identification in Arabic is crucial for almost all NLP tasks, and has recently gained interest among Arabic NLP researchers. One of the early works is that of (Biadsy et al., 2009) where the authors present a system that identifies dialectal words in speech through acoustic signals. Zaidan and Callison-Burch (2011) crawled a large dataset of MSA-DA news commentaries and annotated part of the dataset for sentence-level dialectalness employing Amazon Mechanical Turk. Cotterell and Callison-Burch (2014) extended the previous work by handling more dialects. In (Cotterell et al., 2014), the same authors collect and annotate on Amazon Mechanical Turk a large set of tweets and user commentaries pertaining to five Arabic dialects. Bouamor et al. (2014) select a set of 2,000 Egyptian Arabic sentences and have them translated into four other Arabic dialects to present the first multidialectal Arabic parallel corpus.

Eskander et al. (2014) present a system for handling Arabic written in Roman script "Arabizi". Using decision trees; the system identifies whether each word in the given text is a foreign word or not and further divides non foreign words into four

\footnotetext{
${ }^{1}$ Another group in our lab was responsible for the organization of the task, hence we did not officially participate in the task.
} 
classes: Arabic, Named Entity, punctuation, and sound.

In the context of machine-translation, Salloum and Habash (2011) tackle the problem of DA to English Machine Translation (MT) by pivoting through MSA. The authors present a system that uses DA to MSA transfer rules before applying state of the art MSA to English MT system to produce an English translation. In (Elfardy and Diab, 2012a), we present a set of guidelines for token-level identification of DA while in (Elfardy and Diab, 2012b), (Elfardy et al., 2013) we tackle the problem of token-level dialect-identification by casting it as a code-switching problem. Elfardy and Diab (2013) presents our solution for the sentence-level dialect identification problem.

\section{Shared Task Description}

The shared task for "Language Identification in Code-Switched Data" (Solorio et al., 2014) aims at allowing participants to perform wordlevel language identification in code-switched Spanish-English, MSA-DA, Chinese-English and Nepalese-English data. In this work, we only focus on MSA-DA data. The dataset has six tags:

1. lang1: corresponds to an MSA word, ex. الراهن, AlrAhn ${ }^{2}$ meaning "the current";

2. lang2: corresponds to a DA word, ex. ازيك, ezyk meaning "how are you";

3. mixed: corresponds to a word with mixed morphology, ex. المألوشـون, Alm>lw\$wn meaning "the ones that were excluded or rejected";

4. other: corresponds to punctuation, numbers and words having punctuation or numbers attached to them;

5. ambig: corresponds to a word where the class cannot be determined given the current context, could either be lang1 or lang2; ex. the phrase كله تمام, klh tmAm meaning "all is well" is ambiguous if enough context is not present since it can be used in both MSA and EDA.

6. NE: corresponds to a named-entity, ex. مصر, mSr meaning "Egypt".

\footnotetext{
${ }^{2} \mathrm{We}$ use Buckwalter transliteration scheme http://www.qamus.org/transliteration.htm
}

\section{Approach}

We use a variant of the system that was presented in (Elfardy et al., 2013) to identify the tag of each word in a given Arabic sentence. The original approach relies on language models and a morphological analyzer to assign tags to words in an input sentence. In this new variant, we use MADAMIRA (Pasha et al., 2014); a tool for morphological analysis and disambiguation for Arabic. The advantage of using MADAMIRA over using a morphological analyzer is that MADAMIRA performs contextual disambiguation of the analyses produced by the morphological analyzer, hence reducing the possible options for analyses per word. Figures 1 illustrates the pipeline of the proposed system.

\subsection{Preprocessing}

We experiment with two preprocessing techniques:

1. Basic: In this scheme, we only perform a basic clean-up of the text by separating punctuation and numbers from words, normalizing word-lengthening effects, and replacing all punctuation, URLs, numbers and nonArabic words with $P U N C, U R L, N U M$, and $L A T$ keywords, respectively

2. Tokenized: In this scheme, in addition to basic preprocessing, we use MADAMIRA toolkit to tokenize clitics and affixes by applying the D3-tokenization scheme (Habash and Sadat, 2006). For example, the word بح., bjdwhich means "with seriousness" becomes "ب ج+ جد", "b+ jd” after tokenization.

\subsection{Language Model}

The 'Language Model' (LM) module uses the preprocessed training data to build a 5-gram LM. All tokens in a given sentence in the training data are tagged with either lang1 or lang2 as described in Section 5. The prior probabilities of each lang1 and lang2 words are calculated based on their frequency in the training corpus. SRILM toolkit (Stolcke, 2002) and the tagged corpora are then used to build the LM. ${ }^{3}$ If tokenized preprocessing scheme is used, then the built LM is tokenized where all tokens corresponding to a certain word are assigned the same tag corresponding to the tag

\footnotetext{
${ }^{3} \mathrm{~A}$ full description of the approach is presented in (Elfardy and Diab, 2012b).
} 


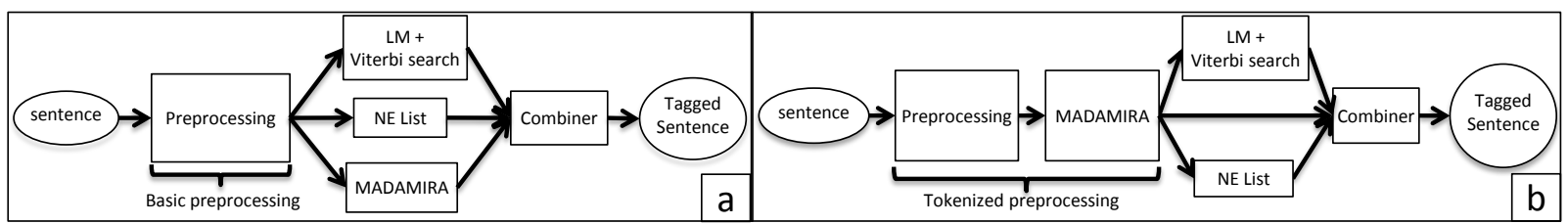

Figure 1: AIDA pipeline. a) The pipeline with the basic preprocessing scheme. b) The pipeline with the tokenized preprocessing scheme.

of the original word. For example, if بح., bjd is tagged as lang2, both "بـد", "جد", jd get tagged as lang2.

For any new untagged sentence, the 'Language Model' module uses the already built LM and the prior probabilities via Viterbi search to find the best sequence of tags for the given sentence. If there is an out-of-vocabulary word in the input sentence, the 'Language Model' leaves it untagged.

\subsection{MADAMIRA}

Using MADAMIRA, each word in a given untagged sentence is tokenized, lemmatized, and POS-tagged. Moreover, the MSA and English glosses for each morpheme of the given word are provided. Since MADAMIRA uses two possible underlying morphological analyzers CALIMA (Habash et al., 2012) and SAMA (Maamouri et al., 2010), as part of the output, MADAMIRA indicates which of them is used to retrieve the glosses.

\subsection{Named Entities List}

We use the ANERGazet (Benajiba et al., 2007) to identify named-entities. ANERGazet consists of the following Gazetteers:

- Locations: 1,545 entries corresponding to names of continents, countries, cities, etc. (ex. المغرب, Almgrb ) which means "Morocco";

- People: 2,100 entries corresponding to names of people. (ex. فهد, fhd);

- Organizations: 318 entries corresponding to names of Organizations such as companies and football teams. (ex. تشلسي, t\$lsy meaning "Chelsea"

\subsection{Combiner}

Each word in the input sentence can get different tags from each module. Thus, the 'Combiner' module uses all of these decisions and the following set of rules to assign the final tag to each word in the input sentence.

1. If the word contains any numbers or punctuation, it is assigned other tag;

2. Else if the word is present in any of the gazetteers or if MADAMIRA assigns it noun_prop POS tag, the word is tagged as $N E$;

3. Else if the word is (or all of its morphemes in the tokenized scheme are) identified by the LM as either lang1 or lang2, the word is assigned the corresponding tag;

4. Else if the word's morphemes are assigned different tags, the word is assigned the mixed tag;

5. Else if the LM does not tag the word (i.e. the word is considered an out of vocabulary word by the LM) and:

- If MADAMIRA retrieved the glosses from SAMA, the word is assigned a lang1 tag;

- Else if MADAMIRA outputs that the glosses were retrieved from CALIMA, then the word is assigned a lang2 tag

- Else if the word is still untagged (i.e. non-analyzable), the word is assigned lang2 tag.

\section{Experiments and Results}

\subsection{Training Phase}

The training data that is used to build our LM consists of two main sources:

1. Shared-task's training data (STT): 119,326 words collected from Twitter. They are manually annotated on the token-level. We split this corpus into:

(a) Training-set; (STT-Tr); 107,398 tweets representing $90 \%$ of $S T T$ and used for training the system 
(b) Development-set; (STT-Dev): 11,928 words representing $10 \%$ of STT and used for tuning the system.

2. Web-log training data (WLT): 8 million words. Half of which comes from langl corpora while the other half is from lang2 corpora. The data is weakly labeled where all tokens in the sentence/comment are assigned the same tag according to the dialect of the forum (MSA or EDA) it was crawled from.

During the development phase, we use STT-Tr and $W L T$ to train our system. We run several experiments to test the different setups and evaluate the performance of each of these setups on STT-Dev. Once we find the optimal configuration, we then use it to retrain the system using all of STT-Tr, STT-Dev, and WLT.

Since the size of $S T T$ is very small compared to $W L T$ ( $0.1 \%$ of $W L T$ size), the existence of six different tags in this corpus can add noise to the already weakly labeled $W L T$ data. Thus, to make STT consistent with $W L T$, we changed the labels of STT as follows:

- If the number of langl tokens in the tweet exceeds the number of lang2 tokens; we assign all tokens in the tweet langl tag.

- Otherwise, all tokens in the tweet are assigned lang2 tag.

All tokens in $S T T$ tagged as $N E$ have been used to enrich our named entity list.

\subsection{Development Phase}

Two different setups are tested using WLT and STT-Tr:

- Surface form setup; uses the basic preprocessing pipeline described earlier on both the input data and on the training data used to build the LM

- Tokenized form setup: uses the tokenized preprocessing pipeline described earlier on both the input data and the training data used to build the LM.

As mentioned earlier, since the size of STT-Tr is much smaller than that of WLT, this causes both datasets to be statistically incomparable. We tried increasing the weights assigned by the LM to STT$T r$ by duplicating STT-Tr. We experimented with one, four, and eight copies of STT-Tr for each of the basic and tokenized experimental setups.

The shared task evaluation script has been used to evaluate each setup. The evaluation script produces two main sets of metrics. The first metric yields the accuracy, precision, recall, and $\mathrm{F}_{\beta=1}$ score for code switching classification on the tweet-level, while the second set of metrics uses evaluates performance of each tag on the tokenlevel. In this paper, we add an extra metric corresponding to the weighted average of the tag on the token level $\mathrm{F}_{\beta=1}$ score in order to rank our overall performance against other participating groups in the task.

Tables 1 and 2 summarize our results for both Surface Form and Tokenized Form setups on STTDev. In all experiments, the Tokenized Form setup outperforms the Surface Form setup.

As shown in Table 2, the system that yields the best weighted-average token-level $\mathrm{F}_{\beta=1}$ score (77.6\%) on the development-set is Tokenized-2. Throughout the rest of the paper, we will use the system's name "AIDA"; to refer to this best configuration (Tokenized-2).

\begin{tabular}{|l|c|c|c|c|}
\hline & Accuracy & Precision & Recall & $\mathbf{F}_{\beta=1}$ \\
\hline Tokenized-1 & $51.5 \%$ & $43.7 \%$ & $97.4 \%$ & $60.3 \%$ \\
\hline Tokenized-2 & $52.5 \%$ & $44.2 \%$ & $97.4 \%$ & $60.8 \%$ \\
\hline Tokenized-8 & $54.2 \%$ & $45.1 \%$ & $96.9 \%$ & $\mathbf{6 1 . 6 \%}$ \\
\hline Surface-1 & $45.4 \%$ & $40.9 \%$ & $99.5 \%$ & $57.9 \%$ \\
\hline Surface-2 & $45.8 \%$ & $41.1 \%$ & $99.5 \%$ & $58.1 \%$ \\
\hline Surface-8 & $46.5 \%$ & $41.4 \%$ & $99.5 \%$ & $58.5 \%$ \\
\hline
\end{tabular}

Table 1: Results on STT-Dev using the tweet-level evaluation. (-1, -2, and -8) correspond to the number of copies of STT-Tr that were added to WLT

\subsection{Testing Phase}

Three blind test sets have been used for the evaluation:

- Test1: 54,732 words of 2,363 tweets collected from some unseen users in the training set;

- Test2: Another 32,641 words of 1,777 tweets collected from other unseen users in the training set;

- Surprise: 12,017 words of 1,222 sentences from collected from Arabic commentaries.

Table 3 shows the distribution of each test set over the different tags 


\begin{tabular}{|l|c|c|c|c|c|c|c|}
\hline & ambig & lang1 & lang2 & mixed & NE & other & Avg-F \\
\hline Tokenized-1 & $0.0 \%$ & $79.5 \%$ & $71.5 \%$ & $0.0 \%$ & $83.6 \%$ & $98.9 \%$ & $77.5 \%$ \\
\hline Tokenized-2 & $0.0 \%$ & $79.6 \%$ & $71.6 \%$ & $0.0 \%$ & $83.6 \%$ & $98.9 \%$ & $\mathbf{7 7 . 6 \%}$ \\
\hline Tokenized-8 & $0.0 \%$ & $79.5 \%$ & $71.4 \%$ & $0.0 \%$ & $83.6 \%$ & $98.9 \%$ & $77.5 \%$ \\
\hline Surface-1 & $0.0 \%$ & $76.0 \%$ & $65.4 \%$ & $0.0 \%$ & $83.6 \%$ & $98.9 \%$ & $73.5 \%$ \\
\hline Surface-2 & $0.0 \%$ & $76.1 \%$ & $65.6 \%$ & $0.0 \%$ & $83.6 \%$ & $98.9 \%$ & $73.7 \%$ \\
\hline Surface-8 & $0.0 \%$ & $76.2 \%$ & $65.5 \%$ & $0.0 \%$ & $83.6 \%$ & $98.9 \%$ & $73.7 \%$ \\
\hline
\end{tabular}

Table 2: Results on STT-Dev using the token-level evaluation. (-1, -2, and -8) correspond to the number of copies of STT-Tr that were added to WLT

\begin{tabular}{|l|c|c|c|c|c|c|}
\hline & ambig & lang1 & lang2 & mixed & NE & other \\
\hline Test1 & $0.0 \%$ & $81.5 \%$ & $0.3 \%$ & $0.0 \%$ & $10.9 \%$ & $7.3 \%$ \\
\hline Test2 & $0.4 \%$ & $32.0 \%$ & $45.3 \%$ & $0.0 \%$ & $13.2 \%$ & $9.0 \%$ \\
\hline Surprise & $0.9 \%$ & $22.4 \%$ & $57.7 \%$ & $0.0 \%$ & $9.1 \%$ & $9.9 \%$ \\
\hline
\end{tabular}

Table 3: Test sets tag distributions

Tables 4, 5, and 6 show the tweet-level evaluation on the three test sets. While tables 7, 8, and 9 show the token-level evaluation on the same test sets. The tables compare the results of our best setup against the other systems that participated in the task ${ }^{4}$.

To make the comparison easier, we have calculated the overall weighted $\mathrm{F}_{\beta=1}$ score for all systems using the three test sets together.

Table 10 shows the $\mathrm{F}_{\beta=1}$ score of each system averaged over all three test-sets. Our system outperforms all other systems in the token-level evaluation and comes in the second place after CMU in the tweet-level classification.

\begin{tabular}{|l|c|c|c|c|}
\hline & Accuracy & Precision & Recall & $\mathbf{F}_{\beta=1}$ \\
\hline AIDA & $45.2 \%$ & $2.3 \%$ & $93.8 \%$ & $4.4 \%$ \\
\hline CMU & $86.1 \%$ & $5.2 \%$ & $53.1 \%$ & $9.5 \%$ \\
\hline A3-107 & $60.5 \%$ & $2.5 \%$ & $71.9 \%$ & $4.8 \%$ \\
\hline IUCL & $97.4 \%$ & $11.1 \%$ & $12.5 \%$ & $11.8 \%$ \\
\hline MSR-IN & $94.7 \%$ & $9.7 \%$ & $34.4 \%$ & $\mathbf{1 5 . 2 \%}$ \\
\hline
\end{tabular}

Table 4: Tweet-level evaluation on Test 1 set.

\begin{tabular}{|l|c|c|c|c|}
\hline & Accuracy & Precision & Recall & $\mathbf{F}_{\beta=1}$ \\
\hline AIDA & $44.0 \%$ & $22.2 \%$ & $95.6 \%$ & $36.0 \%$ \\
\hline CMU & $66.2 \%$ & $29.2 \%$ & $73.4 \%$ & $\mathbf{4 1 . 7 \%}$ \\
\hline A3-107 & $46.9 \%$ & $21.3 \%$ & $82.3 \%$ & $33.8 \%$ \\
\hline IUCL & $76.6 \%$ & $27.1 \%$ & $24.9 \%$ & $26.0 \%$ \\
\hline MSR-IN & $71.4 \%$ & $18.3 \%$ & $21.2 \%$ & $19.6 \%$ \\
\hline
\end{tabular}

Table 5: Tweet-level evaluation on Test2 set.

\footnotetext{
${ }^{4}$ The results of the other groups have been obtained from the workshop website. We use "MSR-IN" to refer to "MSRIndia"
}

\begin{tabular}{|l|c|c|c|c|}
\hline & Accuracy & Precision & Recall & $\mathbf{F}_{\beta=1}$ \\
\hline AIDA & $55.6 \%$ & $16.3 \%$ & $91.2 \%$ & $\mathbf{2 7 . 7 \%}$ \\
\hline CMU & $79.8 \%$ & $20.7 \%$ & $41.2 \%$ & $27.6 \%$ \\
\hline A3-107 & $45.7 \%$ & $12.8 \%$ & $83.3 \%$ & $22.2 \%$ \\
\hline IUCL & $87.7 \%$ & $25.0 \%$ & $15.8 \%$ & $19.4 \%$ \\
\hline MSR-IN & $84.8 \%$ & $17.3 \%$ & $16.7 \%$ & $17.0 \%$ \\
\hline
\end{tabular}

Table 6: Tweet-level evaluation on Surprise set.

\begin{tabular}{|l|c|c|c|c|c|c|c|}
\hline & ambig & lang1 & lang2 & mixed & NE & other & Avg-F \\
$\beta=1$ & \\
\hline AIDA & $0.0 \%$ & $94.5 \%$ & $5.6 \%$ & $0.0 \%$ & $85.0 \%$ & $99.4 \%$ & $\mathbf{9 3 . 6 \%}$ \\
\hline CMU & $0.0 \%$ & $94.4 \%$ & $9.0 \%$ & $0.0 \%$ & $74.0 \%$ & $98.1 \%$ & $92.2 \%$ \\
\hline A3-107 & $0.0 \%$ & $93.8 \%$ & $5.7 \%$ & $0.0 \%$ & $73.4 \%$ & $87.4 \%$ & $90.9 \%$ \\
\hline IUCL & $0.0 \%$ & $88.2 \%$ & $14.2 \%$ & $0.0 \%$ & $0.6 \%$ & $0.6 \%$ & $72.0 \%$ \\
\hline MSR-IN & $0.0 \%$ & $94.2 \%$ & $15.8 \%$ & $0.0 \%$ & $57.7 \%$ & $91.1 \%$ & $89.8 \%$ \\
\hline
\end{tabular}

Table 7: Token-level evaluation on Testl set.

\section{Error Analysis}

Tables 11, 12, and 13 show the confusion matrices of our best setup for all six tags over the three test sets. The rows represent the gold-labels while the columns represent the classes generated by our system. For example, row 4-column 2 corresponds to the percentage of words that have lang 1 (i.e. MSA) gold-label and were incorrectly classified as ambig. The diagonal of each matrix corresponds to the correctly classified instances. All cells of each matrix add-up to $100 \%$. In all three tables, it's clear that the highest confusability is between lang1 and lang2 classes. In Test-set1, since the majority of words $(81.5 \%)$ have a lang 1 gold-label and a very tiny percentage $(0.3 \%)$ has

\begin{tabular}{|l|c|c|c|c|c|c|c|}
\hline & ambig & lang1 & lang2 & mixed & NE & other & Avg-F \\
$\beta=1$ & \\
\hline AIDA & $0.0 \%$ & $73.4 \%$ & $73.2 \%$ & $1.0 \%$ & $91.8 \%$ & $98.1 \%$ & $77.7 \%$ \\
\hline CMU & $0.0 \%$ & $76.3 \%$ & $81.3 \%$ & $0.0 \%$ & $73.4 \%$ & $98.4 \%$ & $\mathbf{7 9 . 9 \%}$ \\
\hline A3-107 & $0.0 \%$ & $62.0 \%$ & $49.4 \%$ & $0.0 \%$ & $67.5 \%$ & $75.0 \%$ & $58.0 \%$ \\
\hline IUCL & $0.0 \%$ & $59.0 \%$ & $59.3 \%$ & $0.0 \%$ & $13.1 \%$ & $1.7 \%$ & $47.7 \%$ \\
\hline MSR-IN & $1.5 \%$ & $58.7 \%$ & $50.5 \%$ & $0.0 \%$ & $42.4 \%$ & $43.8 \%$ & $51.3 \%$ \\
\hline
\end{tabular}

Table 8: Token-level evaluation on Test 2 set. 


\begin{tabular}{|l|c|c|c|c|c|c|c|}
\hline & ambig & lang1 & lang2 & mixed & NE & other & Avg-F \\
$\beta=1$ \\
\hline AIDA & $0.0 \%$ & $66.6 \%$ & $81.9 \%$ & $0.0 \%$ & $87.9 \%$ & $99.9 \%$ & $\mathbf{8 0 . 1} \%$ \\
\hline CMU & $0.0 \%$ & $68.0 \%$ & $82.1 \%$ & $0.0 \%$ & $61.2 \%$ & $97.5 \%$ & $77.8 \%$ \\
\hline A3-107 & $0.0 \%$ & $53.8 \%$ & $61.3 \%$ & $0.0 \%$ & $62.3 \%$ & $96.1 \%$ & $62.6 \%$ \\
\hline IUCL & $0.0 \%$ & $48.8 \%$ & $60.9 \%$ & $0.0 \%$ & $5.5 \%$ & $2.0 \%$ & $46.7 \%$ \\
\hline MSR-IN & $0.0 \%$ & $56.3 \%$ & $69.8 \%$ & $0.0 \%$ & $33.2 \%$ & $96.6 \%$ & $65.4 \%$ \\
\hline
\end{tabular}

Table 9: Token-level evaluation on Surprise set.

\begin{tabular}{|l|c|c|}
\hline & Tweet Avg-F $_{\beta=1}$ & Token Avg-F $_{\beta=1}$ \\
\hline AIDA & $20.2 \%$ & $\mathbf{8 6 . 8 \%}$ \\
\hline CMU & $\mathbf{2 4 . 3 \%}$ & $86.4 \%$ \\
\hline A3-107 & $18.4 \%$ & $76.6 \%$ \\
\hline IUCL & $18.2 \%$ & $61.0 \%$ \\
\hline MSR-IN & $17.1 \%$ & $74.2 \%$ \\
\hline
\end{tabular}

Table 10: Overall tweet-level and token-level $\mathrm{F}_{\beta=1}$ scores. (Averaged over the three test-sets)

a lang2 gold-label, the percentage of words that have a gold label of langl and get classified as lang2 is much larger than in the other two test-sets and much larger than the opposite-case where the ones having a gold-label of lang2 get classified as lang1.

Table 14 shows examples of the words that were misclassified by AIDA. All of the shown examples are quite challenging. In example 1, the misclassified named-entity refers to the name of a TV show but the word also means "clearly" which is a "lang1" word. Similarly in example 2, the namedentity can mean "stable" which is again a "langl" word. Another misclassification is that in example 3, where a mixed-morphology "mixed" word meaning "those who were excluded/rejected" is misclassified as being a "lang2" word. When we looked at why this happened, we found that the word wasn't tokenized by MADAMIRA. Our approach only assigns "mixed" tag if after tokenization, different morphemes of the word get different tags. Since in this example the word wasn't tokenized, it could not get the "mixed" tag. However, "lang2" tag (assigned by AIDA) is the second most appropriate tag since the main morpheme of the word is dialectal/lang2. An example of a "mixed" word that was correctly classified by AIDA is حتوءدي, Ht\&dy meaning "will lead to" where the main morpheme توءsd, t\&dy "lead to" is "lang1" and the clitic ح, $\mathrm{H}$ "will" is "lang2".

Examples 4 and 5 show instances of the confusability between "langl" and "lang2" classes. Both words in these two examples can belong to either one of "langl" and "lang2" classes depending on the context.

One interesting observation is that AIDA, outperforms all other systems tagging named-entities. This suggests the robustness of the NER approach used by AIDA.

The performance on the other tags varies across the three test-sets.

\begin{tabular}{|c|c|c|c|c|c|c|}
\hline & \multicolumn{6}{|c|}{ AIDA (Predicted) } \\
\hline & ambig & lang1 & lang2 & mixed & NE & other \\
\hline ambig & $0.0 \%$ & $0.0 \%$ & $0.0 \%$ & $0.0 \%$ & $0.0 \%$ & $0.0 \%$ \\
\hline lang1 & $0.0 \%$ & $74.4 \%$ & $5.7 \%$ & $0.0 \%$ & $1.3 \%$ & $0.0 \%$ \\
\hline lang2 & $0.0 \%$ & $0.1 \%$ & $0.2 \%$ & $0.0 \%$ & $0.0 \%$ & $0.0 \%$ \\
\hline mixed & $0.0 \%$ & $0.0 \%$ & $0.0 \%$ & $0.0 \%$ & $0.0 \%$ & $0.0 \%$ \\
\hline NE & $0.0 \%$ & $1.5 \%$ & $0.3 \%$ & $0.0 \%$ & $9.1 \%$ & $0.1 \%$ \\
\hline other & $0.0 \%$ & $0.0 \%$ & $0.0 \%$ & $0.0 \%$ & $0.0 \%$ & $7.3 \%$ \\
\hline
\end{tabular}

Table 11: The token-level confusion matrix for the best performing setup on Test 1 set.

\begin{tabular}{|l|c|c|c|c|c|c|}
\hline & \multicolumn{6}{|c|}{ AIDA (Predicted) } \\
\hline & ambig & lang1 & lang2 & mixed & NE & other \\
\hline ambig & $0.0 \%$ & $0.3 \%$ & $0.1 \%$ & $0.0 \%$ & $0.0 \%$ & $0.0 \%$ \\
\hline lang1 & $0.0 \%$ & $28.8 \%$ & $2.8 \%$ & $0.1 \%$ & $0.2 \%$ & $0.1 \%$ \\
\hline lang2 & $0.0 \%$ & $16.4 \%$ & $28.3 \%$ & $0.5 \%$ & $0.2 \%$ & $0.1 \%$ \\
\hline mixed & $0.0 \%$ & $0.0 \%$ & $0.0 \%$ & $0.0 \%$ & $0.0 \%$ & $0.0 \%$ \\
\hline NE & $0.0 \%$ & $1.0 \%$ & $0.6 \%$ & $0.0 \%$ & $11.5 \%$ & $0.2 \%$ \\
\hline other & $0.0 \%$ & $0.0 \%$ & $0.0 \%$ & $0.0 \%$ & $0.0 \%$ & $8.9 \%$ \\
\hline
\end{tabular}

Table 12: The token-level confusion matrix for the best performing setup on Test 2 set.

\begin{tabular}{|l|c|c|c|c|c|c|}
\hline & \multicolumn{6}{|c|}{ AIDA (Predicted) } \\
\hline & ambig & lang1 & lang2 & mixed & NE & other \\
\hline ambig & $0.0 \%$ & $0.6 \%$ & $0.3 \%$ & $0.0 \%$ & $0.0 \%$ & $0.0 \%$ \\
\hline lang1 & $0.0 \%$ & $19.0 \%$ & $2.9 \%$ & $0.0 \%$ & $0.5 \%$ & $0.0 \%$ \\
\hline lang2 & $0.0 \%$ & $14.5 \%$ & $42.7 \%$ & $0.0 \%$ & $0.5 \%$ & $0.0 \%$ \\
\hline mixed & $0.0 \%$ & $0.0 \%$ & $0.0 \%$ & $0.0 \%$ & $0.0 \%$ & $0.0 \%$ \\
\hline NE & $0.0 \%$ & $0.5 \%$ & $0.6 \%$ & $0.0 \%$ & $8.0 \%$ & $0.0 \%$ \\
\hline other & $0.0 \%$ & $0.0 \%$ & $0.0 \%$ & $0.0 \%$ & $0.0 \%$ & $9.9 \%$ \\
\hline
\end{tabular}

Table 13: The token-level confusion matrix for the best performing setup on Surprise set. 


\begin{tabular}{|c|c|c|c|c|}
\hline & Sentence & Word & Gold-Label & AIDA-Label \\
\hline Ex. 1. & 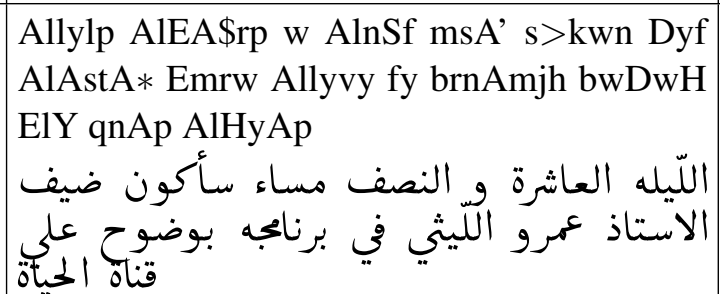 & bwDwH, بوضوح & $\mathrm{NE}$ & lang1 \\
\hline Ex. 2. & 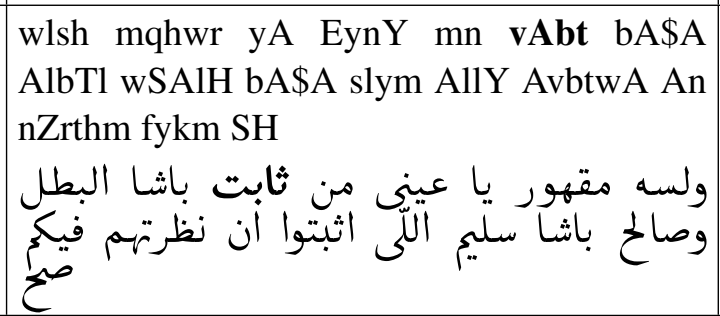 & vAbt, ثابت & $\mathrm{NE}$ & lang1 \\
\hline Ex. 3. & $\begin{array}{l}\text { Anh tAnY yqwm hykwn mE Alm>lw\$yn } \\
\text { انه تانى يقوم هيكون مع المألوثين }\end{array}$ & Alm>lw\$yn, المألوشين & mixed & lang2 \\
\hline Ex. 4. & 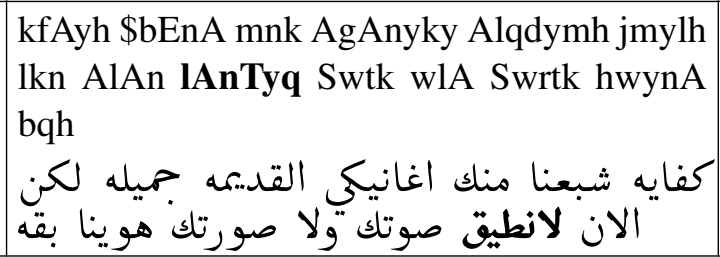 & لانطيق 1AnTyq & lang1 & lang2 \\
\hline Ex. 5. & 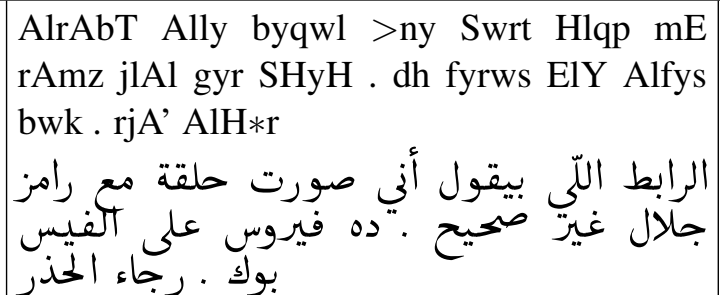 & ملقة Hlqp, & lang2 & lang1 \\
\hline
\end{tabular}

Table 14: Examples of the words that were misclassified by AIDA

\section{Conclusion and Future Work}

In this work, we adapt a previously proposed system for automatic detection of code switching in informal Arabic text to handle twitter data. We experiment with several setups and report the results on two twitter datasets and a surprise-genre test-set, all of which were generated for the shared task at EMNLP workshop for Computational Approaches to Code Switching. In the future we plan on handling other Arabic dialects such as Levantine, Iraqi and Moroccan Arabic as well as adapting the system to other genres.

\section{Acknowledgment}

This work has been funded by the NSF CRI Code Switching Project.

We would like to thank Mahmoud Ghoneim for his thorough review of the paper and the data. We also thank the anonymous reviewer for the useful comments.

\section{References}

Yassine Benajiba, Paolo Rosso, and Jos Miguel Benedruiz. 2007. Anersys: An arabic named entity recognition system based on maximum entropy. In In Proceedings of CICLing-2007.

Fadi Biadsy, Julia Hirschberg, and Nizar Habash. 2009. Spoken arabic dialect identification using phonotactic modeling. In Proceedings of the Workshop on Computational Approaches to Semitic Languages at the meeting of the European Association for Computational Linguistics (EACL), Athens, Greece.

Houda Bouamor, Nizar Habash, and Kemal Oflazer. 2014. A multidialectal parallel corpus of arabic. In Proceedings of LREC.

Ryan Cotterell and Chris Callison-Burch. 2014. A multi-dialect, multi-genre corpus of informal written 
arabic. In Proceedings of the Language Resources and Evaluation Conference (LREC).

Ryan Cotterell, Adithya Renduchintala, Naomi Saphra, and Chris Callison-Burch. 2014. An algerian arabic-french code-switched corpus. In LREC Workshop on Free/Open-Source Arabic Corpora and Corpora Processing Tools.

Heba Elfardy and Mona Diab. 2012a. Simplified guidelines for the creation of large scale dialectal arabic annotations. In Proceedings of LREC.

Heba Elfardy and Mona Diab. 2012b. Token level identification of linguistic code switching. In Proceedings of COLING, Mumbai, India.

Heba Elfardy and Mona Diab. 2013. Sentence-Level Dialect Identification in Arabic. In Proceedings of ACL2013, Sofia, Bulgaria, August.

Heba Elfardy, Mohamed Al-Badrashiny, and Mona Diab. 2013. Code Switch Point Detection in Arabic. In Proceedings of the 18th International Conference on Application of Natural Language to Information Systems (NLDB2013), MediaCity, UK, June.

Ramy Eskander, Mohamed Al-Badrashiny, Nizar Habash, and Owen Rambow. 2014. Foreign words and the automatic processing of arabic social media text written in roman script. In Proceedings of the First Workshop on Computational Approaches to Code-Switching. EMNLP 2014, Conference on Empirical Methods in Natural Language Processing, October, 2014, Doha, Qatar.

Ferguson. 1959. Diglossia. Word 15. 325340.

Nizar Habash and Fatiha Sadat. 2006. Arabic preprocessing schemes for statistical machine translation.

Nizar Habash, Ramy Eskander, and AbdelAti Hawwari. 2012. A Morphological Analyzer for Egyptian Arabic. In NAACL-HLT 2012 Workshop on Computational Morphology and Phonology (SIGMORPHON2012), pages 1-9.

Nizar Habash. 2010. Introduction to arabic natural language processing. Advances in neural information processing systems.

Mohamed Maamouri, Dave Graff, Basma Bouziri, Sondos Krouna, Ann Bies, and Seth Kulick. 2010. Ldc standard arabic morphological analyzer (sama) version 3.1.

Arfath Pasha, Mohamed Al-Badrashiny, Mona Diab, Ahmed El Kholy, Ramy Eskander, Nizar Habash, Manoj Pooleery, Owen Rambow, and Ryan M. Roth. 2014. MADAMIRA: A Fast, Comprehensive Tool for Morphological Analysis and Disambiguation of Arabic. In Proceedings of LREC, Reykjavik, Iceland.
Wael Salloum and Nizar Habash. 2011. Dialectal to standard arabic paraphrasing to improve arabicenglish statistical machine translation. In Proceedings of the First Workshop on Algorithms and Resources for Modelling of Dialects and Language Varieties. Association for Computational Linguistics.

Thamar Solorio, Elizabeth Blair, Suraj Maharjan, Steve Bethard, Mona Diab, Mahmoud Gonheim, Abdelati Hawwari, Fahad AlGhamdi, Julia Hirshberg, Alison Chang, , and Pascale Fung. 2014. Overview for the first shared task on language identification in code-switched data. In In Proceedings of the First Workshop on Computational Approaches to CodeSwitching. EMNLP 2014, Conference on Empirical Methods in Natural Language Processing, October, 2014, Doha, Qatar.

Andreas Stolcke. 2002. Srilm an extensible language modeling toolkit. In Proceedings of ICSLP.

Omar F Zaidan and Chris Callison-Burch. 2011. The arabic online commentary dataset: an annotated dataset of informal arabic with high dialectal content. In $A C L$. 